PROCEEDINGS OF THE AMERICAN MATHEMATICAL SOCIETY

Volume 124, Number 8, August 1996

\title{
BAIRE SPACES AND HYPERSPACE TOPOLOGIES
}

\author{
LÁSZLÓ ZSILINSZKY \\ (Communicated by Franklin D. Tall)
}

\begin{abstract}
Sufficient conditions for abstract (proximal) hit-and-miss hyperspace topologies and the Wijsman hyperspace topology, respectively, are given to be Baire spaces, thus extending results of McCoy, Beer, and Costantini. Further the quasi-regularity of (proximal) hit-and-miss topologies is investigated.
\end{abstract}

\section{INTRODUCTION}

Recently there has been considerable interest in investigating properties of socalled hit-and-miss, resp. proximal hit-and-miss hyperspace topologies, i.e. topologies on the class $C L(X)$ of all nonempty closed subsets of a topological, resp. uniform space $X$ (see [B2], [BT1], [BT2], [DMH], [V]). To describe these topologies, for any $E \subset X$ denote $E^{-}=\{A \in C L(X) ; A \cap E \neq \emptyset\}, E^{+}=\{A \in C L(X) ; A \subset E\}$. Further if $(X, \mathcal{U})$ is a uniform space, put $E^{++}=\{A \in C L(X) ; \exists U \in \mathcal{U}$ with $U[A] \subset$ $E\}$, where $U[A]=\{x \in X ; \exists a \in A$ with $(x, a) \in U\}$. Sets in $E^{-}$"hit" $E$, whereas sets in $E^{+}$"miss" the complement $E^{c}$ of $E$ and sets in $E^{++}$are "far" from $E^{c}$. The abstract hit-and-miss topology for $C L(X)$ (first studied by Poppe in [P1], [P2]) has as a subbase all sets of the form $V^{-}$, where $V$ is an arbitrary open subset of $X$ plus all sets of the form $\left(B^{c}\right)^{+}$, where $B$ ranges over a given nonempty family $\Delta \subset C L(X)$. If $(X, \mathcal{U})$ is a uniform space and $\left(B^{c}\right)^{+}$is replaced by $\left(B^{c}\right)^{++}$in the above definition we get the so-called proximal hit-and-miss topology (cf. [B2]). Varying $\Delta$ we obtain diverse hyperspace topologies: if $\Delta=C L(X)$ the familiar Vietoris topology $\tau_{V}$ (cf. $[\mathrm{KT}],[\mathrm{Mi}]$ ), resp. the proximal Vietoris topology $\tau_{p V}$ (cf. [DMN], [BLLN]); if $\Delta=$ nonempty closed compact subsets of $X$, the Fell topology $\tau_{F}$ (cf. $[\mathrm{F}],[\mathrm{KT}]$ ), resp. the proximal Fell topology $\tau_{p F}$ (this clearly coincides with $\tau_{F}$ in case of a Hausdorff space $X$ ); if $\Delta=$ closed proper balls in a metric space, the ball topology $\tau_{B}$, resp. ball-proximal topology $\tau_{p B}$ (cf. [HL], [BT1]). For other topologies see [B2].

If $(X, d)$ is a metric space we will consider one more hyperspace topology, the socalled Wijsman topology $\tau_{W}$, which is the weak topology generated by the distance functionals $d(x, A)=\inf \{d(x, a) ; a \in A\}$ (where $x \in X, \emptyset \neq A \subset X$ ) viewed as functionals of set argument (see [FLL], [B2]). Although this topology is neither

Received by the editors February 1, 1995.

1991 Mathematics Subject Classification. Primary 54B20; Secondary 54E52.

Key words and phrases. Baire space, complete space, quasi-regular space, (proximal) hit-andmiss topology, (proximal) Vietoris topology, (proximal) Fell topology, ball and ball-proximal topology, Wijsman topology.

(C)1996 American Mathematical Society 
hit-and-miss nor proximal hit-and-miss in general (cf. [HL]), it is a fundamental tool in the construction of the lattice of hyperspace topologies, for most of the above and many other known topologies arise as suprema and infima, respectively, of appropriate Wijsman topologies (cf. [BLLN], [CLP]).

The question as to when the hyperspace is a Baire space, i.e. a space where every countable intersection of dense open subsets is dense, has been thoroughly investigated by McCoy in $[\mathrm{MC}]$ for the Vietoris topology. For other hyperspace topologies there are only partial results, e.g. if $X$ is a locally compact Hausdorff space, then $\left(C L(X), \tau_{F}\right)$ is a locally compact Hausdorff space $([\mathrm{KT}])$, hence a Baire space as well; or if $X$ is metrizable with a separable complete metric, then $\left(C L(X), \tau_{W}\right)$ is completely metrizable $([\mathrm{B} 1],[\mathrm{C}])$ and is thus a Baire space. It is the purpose of this paper to find sufficient conditions for the Baireness of abstract (proximal) hitand-miss topologies and as a consequence also for the Wijsman topology. We will make use of $[\mathrm{MC}]$, properly modifying and extending its techniques and ideas. In order to achieve this we first find conditions for the quasi-regularity of (proximal) hit-and-miss topologies. It turns out that $\left(C L(X), \tau_{p V}\right)$ is a Baire space if $X$ is e.g. a Baire uniform space with a countable pseudo-base; $\left(C L(X), \tau_{F}\right)$ is a Baire space if $X$ is almost locally compact and nonempty closed compact subsets of $X$ have open neighbourhoods with compact closure; $\left(C L(X), \tau_{p F}\right)$ is a Baire space if $X$ is a locally compact uniform space; and finally $C L(X)$ endowed with $\tau_{B}, \tau_{p B}$, and $\tau_{W}$, respectively is a Baire space if $X$ is a separable Baire metric space or a completely metrizable space.

\section{Notation AND TERMinOLOGY}

Let $(X, \tau)$ be a topological space and $\omega$ stand for the set of all positive integers. Let $C L(X), K(X)$ be respectively the nonempty closed and nonempty closed compact subsets of $X$. If $E \subset X$, then $\bar{E}$, int $E$ will stand for the closure and interior, respectively, of $E$ in $X$. In a metric space $(X, d)$ denote by $S(x, r)(B(x, r))$ the open (closed) ball about $x$ of radius $r>0$ and write $B(X)$ for the nonempty closed proper balls of $X$. Given nonempty sets $A, B \subset X$ define the gap between them as $D(A, B)=\inf \{d(a, b) ; a \in A, b \in B\}$. Throughout the paper when speaking of notions concerning uniform spaces we will assume that $X$ is endowed with a uniformity $\mathcal{U}$. A topological space $X$ is said to be $R_{0}$ (pseudo- $\left.R_{0}\right)$ if every nonempty open subset of $X$ contains the closure of each (of some) of its points (cf. [D], [Zs]); further $X$ is called quasi-regular if every nonempty open set contains a closed set with nonempty interior.

A collection $\mathcal{P} \subset \tau \backslash\{\emptyset\}$ is said to be a pseudo-base for $X$ if every nonempty $\tau$-open set contains a member of $\mathcal{P}(\mathrm{cf}$. $[\mathrm{O}])$. Define the following sets $([\mathrm{MC}])$ :

$$
\begin{gathered}
S(X, \mathcal{P})=\{f: \mathcal{P} \rightarrow \mathcal{P} ; f(U) \subset U \text { for every } U \in \mathcal{P}\} \text { and } \\
R S(X, \mathcal{P})=\{f: \mathcal{P} \rightarrow \mathcal{P} ; \overline{f(U)} \subset U \text { for every } U \in \mathcal{P}\} .
\end{gathered}
$$

If $U \in \mathcal{P}$ and $f, g \in S(X, \mathcal{P})$ or $R S(X, \mathcal{P})$, define $[U, f, g]_{1}=g(U)$ and for $i>1$

$$
[U, f, g]_{i}= \begin{cases}f\left([U, f, g]_{i-1}\right), & \text { if } i \text { is even, } \\ g\left([U, f, g]_{i-1}\right), & \text { if } i \text { is odd. }\end{cases}
$$

Write $\left(U_{1}, \ldots, U_{n}\right)_{B}^{+}$for $\left(B^{c}\right)^{+} \cap \bigcap_{i=1}^{n} U_{i}^{-} \subset C L(X)$, further $\left(U_{1}, \ldots, U_{n}\right)_{B}^{++}$for $\left(B^{c}\right)^{++} \cap \bigcap_{i=1}^{n} U_{i}^{-}$and $\left(U_{1}, \ldots, U_{n}\right)_{B}^{*}$ for $\prod_{i=1}^{n}\left(B^{c} \cap U_{i}\right) \times \prod_{i=n+1}^{\infty} B^{c} \subset X^{\omega}$, where $B, U_{1}, \ldots, U_{n} \subset X(n \in \omega)$. Then for any $\mathbf{U}=\left(U_{1}, \ldots, U_{n}\right)_{B}^{+}$(resp. 
$\left.\mathbf{U}=\left(U_{1}, \ldots, U_{n}\right)_{B}^{++}\right)$we can assign $\mathbf{U}_{*}=\left(U_{1}, \ldots, U_{n}\right)_{B}^{*}$ and conversely for any $\mathbf{U}=$ $\left(U_{1}, \ldots, U_{n}\right)_{B}^{*}$ we can put $\mathbf{U}_{+}=\left(U_{1}, \ldots, U_{n}\right)_{B}^{+}\left(\right.$resp. $\left.\mathbf{U}_{++}=\left(U_{1}, \ldots, U_{n}\right)_{B}^{++}\right)$. In what follows $\Delta$ will be a fixed nonempty subfamily of $C L(X)$ and $\Delta_{0}=\Delta \cup\{\emptyset\}$. For $\Delta^{\prime} \subset \Delta_{0}$ denote by $\Sigma\left(\Delta^{\prime}\right)$ the set of all finite unions of members of $\Delta^{\prime}$. Then $\mathcal{B}_{\Delta}^{*}=\left\{\left(U_{1}, \ldots, U_{n}\right)_{B}^{*} ; B \in \Sigma\left(\Delta_{0}\right), U_{1}, \ldots, U_{n} \in \tau, n \in \omega\right\}$ is a base for a topology $\tau_{\Delta}^{*}$ on $X^{\omega}$. Indeed, if $\mathbf{U}=\left(U_{1} \ldots, U_{n}\right)_{B}^{*}, \mathbf{V}=\left(V_{1}, \ldots, V_{m}\right)_{D}^{*} \in \mathcal{B}_{\Delta}^{*}$ and $n \geq m$, then

$$
\mathbf{U} \cap \mathbf{V}=\left(U_{1} \cap V_{1}, \ldots, U_{m} \cap V_{m}, U_{m+1}, \ldots, U_{n}\right)_{B \cup D}^{*} \in \mathcal{B}_{\Delta}^{*} .
$$

The hit-and-miss topology (resp. proximal hit-and-miss topology) $\tau_{\Delta}^{+}$(resp. $\tau_{\Delta}^{++}$) on $C L(X)$ has as a base $\mathcal{B}_{\Delta}^{+}$(resp. $\mathcal{B}_{\Delta}^{++}$) all sets of the form $\left(U_{1}, \ldots, U_{n}\right)_{B}^{+}$(resp. $\left.\left(U_{1}, \ldots, U_{n}\right)_{B}^{++}\right)$, where $B \in \Sigma\left(\Delta_{0}\right), U_{1}, \ldots, U_{n} \in \tau, n \in \omega$. The symbols $c l_{*}(\mathbf{E})$, $c l_{+}(\mathbf{E})$ and $c l_{++}(\mathbf{E})$ will stand for the closure of $\mathbf{E}$ in $\left(X^{\omega}, \tau_{\Delta}^{*}\right),\left(C L(X), \tau_{\Delta}^{+}\right)$and $\left(C L(X), \tau_{\Delta}^{++}\right)$, respectively. Whenever $f: \mathcal{B}_{\Delta}^{*} \rightarrow \mathcal{B}_{\Delta}^{*}$, define $f_{+}(\mathbf{U})=\left(f\left(\mathbf{U}_{*}\right)\right)_{+}$ $\left(f_{++}(\mathbf{U})=\left(f\left(\mathbf{U}_{*}\right)\right)_{++}\right)$for every $\mathbf{U} \in \mathcal{B}_{\Delta}^{+}\left(\mathbf{U} \in \mathcal{B}_{\Delta}^{++}\right)$and conversely whenever $f: \mathcal{B}_{\Delta}^{+} \rightarrow \mathcal{B}_{\Delta}^{+}\left(f: \mathcal{B}_{\Delta}^{++} \rightarrow \mathcal{B}_{\Delta}^{++}\right)$define $f_{*}(\mathbf{U})=\left(f\left(\mathbf{U}_{+}\right)\right)_{*}\left(f_{*}(\mathbf{U})=\left(f\left(\mathbf{U}_{++}\right)\right)_{*}\right)$ for every $\mathbf{U} \in \mathcal{B}_{\Delta}^{*}$. A mapping $f$ from a topological space $X$ onto a topological space $Y$ is said to be feebly continuous (feebly open) if $\operatorname{int} f^{-1}(V) \neq \emptyset(\operatorname{int} f(U) \neq \emptyset)$ for any nonempty open $V \subset Y(U \subset X)$. A feeble homeomorphism is a feebly continuous feebly open bijection (cf. [HMC]).

\section{Auxiliary Results}

The following characterization of Baire spaces is proved in $[\mathrm{MC}]$ (Theorem 2.3).

Theorem 2.1. Let $X$ be a quasi-regular space and $\mathcal{P}$ a pseudo-base for $X$. Then $X$ is a Baire space if and only if for every $U \in \mathcal{P}$ and $f \in R S(X, \mathcal{P})$ there exists $g \in S(X, \mathcal{P})$ such that $\bigcap_{i=1}^{\infty}[U, f, g]_{i} \neq \emptyset$.

Lemma 2.2. (i) Suppose that $X$ has a countable pseudo-base and there exists a countable family $\Delta^{\prime} \subset \Delta$ such that whenever $B \in \Sigma(\Delta), B \neq X$ and $W_{i} \in \tau \backslash\{\emptyset\}$ are disjoint for each $1 \leq i \leq n(n \in \omega)$ there exists $D \in \Sigma\left(\Delta^{\prime}\right)$ such that $B \subset D \subsetneq X$ and $W_{i} \cap D^{c} \neq \emptyset$ for every $1 \leq i \leq n$. Then $\left(X^{\omega}, \tau_{\Delta}^{*}\right)$ has a countable pseudo-base.

(ii) If $X$ is a Baire space and $\left(X^{\omega}, \tau_{\Delta}^{*}\right)$ has a countable pseudo-base, then $\left(X^{\omega}, \tau_{\Delta}^{*}\right)$ is a Baire space.

Proof. (i) Let $\mathcal{P}$ be a countable pseudo-base for $X$. Then $\left\{\left(U_{1}, \ldots, U_{n}\right)_{B}^{*} ; B \in\right.$ $\left.\Sigma\left(\Delta^{\prime} \cup\{\emptyset\}\right), U_{1}, \ldots, U_{n} \in \mathcal{P}, n \in \omega\right\}$ forms a countable pseudo-base for $X^{\omega}$. As for (ii), it can be shown analogously to the proof of Theorem 2.6 in [O]. The only difference is that instead of the sequence $\left\{Y^{\left(n_{k}\right)}\right\}_{k}$ we should consider a sequence $B_{k}^{\left(n_{k}\right)}=\prod_{j>n_{k}} B_{k}^{c}$ for some $B_{k} \in \Sigma\left(\Delta_{0}\right)(k \in \omega)$.

Lemma 2.3. Let $B \in \Sigma\left(\Delta_{0}\right)$ and $U_{1}, \ldots, U_{n} \in \tau(n \in \omega)$. Then

(i) $c l_{*}\left(\left(U_{1}, \ldots, U_{n}\right)_{B}^{*}\right)=\left(\overline{U_{1} \cap B^{c}}, \ldots, \overline{U_{n} \cap B^{c}}\right)_{i n t B}^{*}$;

(ii) if $X$ is pseudo- $R_{0}$, then $c l_{+}\left(\left(U_{1}, \ldots, U_{n}\right)_{B}^{+}\right) \supset\left(\overline{U_{1} \cap B^{c}}, \ldots, \overline{U_{n} \cap B^{c}}\right)_{\text {intB }}^{+}$;

(iii) $c l_{++}\left(\left(U_{1}, \ldots, U_{n}\right)_{B}^{++}\right) \supset\left(\overline{U_{1} \cap B^{c}}, \ldots, \overline{U_{n} \cap B^{c}}\right)_{\text {int } B}^{+}$.

Proof. (i) This follows from the definition of closure in $(X, \tau)$ and $\left(X^{\omega}, \tau_{\Delta}^{*}\right)$, respectively.

(ii) The proof of Hilfsatz $5(\mathrm{~b})$ in [P2] works for pseudo- $R_{0}$ spaces as well if point-closures are used instead of singletons. 
(iii) Let $A \in\left(\overline{U_{1} \cap B^{c}}, \ldots, \overline{U_{n} \cap B^{c}}\right)_{i n t B}^{+}$and denote $\mathbf{U}=\left(U_{1}, \ldots, U_{n}\right)_{B}^{++}$. Let $\mathbf{V}=\left(V_{1}, \ldots, V_{m}\right)_{D}^{++}$be a $\tau_{\Delta}^{++}$-neighbourhood of $A$. Then $U[A] \cap U[D]=\emptyset$ for some $U \in \mathcal{U}$. It is not hard to find $a_{i} \in U[A] \cap U_{i} \cap B^{c}$ and $b_{j} \in U[A] \cap V_{j} \cap B^{c}$ for each $1 \leq i \leq n$ and $1 \leq j \leq m$. Then there exist entourages $W_{i}$ and $Z_{j}$ with $a_{i} \in W_{i}[B]^{c}$ and $b_{j} \in Z_{j}[B]^{c}$ for all $1 \leq i \leq n$ and $1 \leq j \leq m$. Put $V=U \cap \bigcap_{i=1}^{n} W_{i} \cap \bigcap_{j=1}^{m} Z_{j} \in \mathcal{U}$. Then $A_{0}=\bigcup_{i=1}^{n} \overline{\left\{a_{i}\right\}} \cup \bigcup_{j=1}^{m} \overline{\left\{b_{j}\right\}} \subset V[B]^{c}$, so $A_{0} \in\left(B^{c}\right)^{++}$; consequently $A_{0} \in \mathbf{U} \cap \mathbf{V}$, whence $A \in c l_{++}(\mathbf{U})$.

Lemma 2.4. Let $B, D \in \Sigma\left(\Delta_{0}\right)$ and $U_{1}, \ldots, U_{n}, V_{1}, \ldots, V_{m} \in \tau(m, n \in \omega)$. Then $\left(\overline{V_{1} \cap D^{c}}, \ldots, \overline{V_{m} \cap D^{c}}\right)_{\text {intD }}^{+} \subset\left(U_{1}, \ldots, U_{n}\right)_{B}^{+} \quad$ (resp. $\left(\overline{V_{1} \cap D^{c}}, \ldots, \overline{V_{m} \cap D^{c}}\right)_{i n t D}^{+} \subset$ $\left.\left(U_{1}, \ldots, U_{n}\right)_{B}^{++}\right)$implies that $\overline{D^{c}} \subset B^{c}$ and for all $1 \leq i \leq n$ there exists $1 \leq j \leq m$ such that $\overline{V_{j} \cap D^{c}} \subset U_{i}$.

Proof. Let $A \in\left(\overline{V_{1} \cap D^{c}}, \ldots, \overline{V_{m} \cap D^{c}}\right)_{i n t D}^{+}=\mathbf{V}$ and denote $\mathbf{U}=\left(U_{1}, \ldots, U_{n}\right)_{B}^{+}$. Suppose that $A_{0}=\overline{D^{c}} \cap B \neq \emptyset$. Then $A \cup A_{0} \in \mathbf{V} \backslash \mathbf{U}$, which is a contradiction; thus $\overline{D^{c}} \subset B^{c}$. Similarly, if there exists an $1 \leq i \leq n$ such that $A_{j}=\overline{V_{j} \cap D^{c}} \backslash U_{i} \neq \emptyset$ for each $1 \leq j \leq m$, then $\bigcup_{j=1}^{m} A_{j} \in \mathbf{V} \backslash \mathbf{U}$, and the proof follows. As for the statement in parentheses observe that always $\left(U_{1}, \ldots, U_{n}\right)_{B}^{++} \subset\left(U_{1}, \ldots, U_{n}\right)_{B}^{+}$.

Lemma 2.5. We have

(i) $f_{+} \in S\left(C L(X), \mathcal{B}_{\Delta}^{+}\right) \quad\left(f_{++} \in S\left(C L(X), \mathcal{B}_{\Delta}^{++}\right)\right)$for every $f \in S\left(X^{\omega}, \mathcal{B}_{\Delta}^{*}\right)$;

(ii) if $X$ is pseudo- $R_{0}$, then $f_{*} \in R S\left(X^{\omega}, \mathcal{B}_{\Delta}^{*}\right)$ for each $f \in R S\left(C L(X), \mathcal{B}_{\Delta}^{+}\right)$,

(iii) $f_{*} \in R S\left(X^{\omega}, \mathcal{B}_{\Delta}^{*}\right)$ for each $f \in R S\left(C L(X), \mathcal{B}_{\Delta}^{++}\right)$.

Proof. Only (ii) and (iii) need some comments. Let $f \in R S\left(C L(X), \mathcal{B}_{\Delta}^{+}\right)$and denote $\mathbf{U}=\left(U_{1}, \ldots, U_{n}\right)_{B}^{*} \in \mathcal{B}_{\Delta}^{*}$. Then $f\left(\mathbf{U}_{+}\right)=\left(V_{1}, \ldots, V_{m}\right)_{D}^{+} \in \mathcal{B}_{\Delta}^{+}$and by Lemma 2.3 (ii) we get $\left(\overline{V_{1} \cap D^{c}}, \ldots, \overline{V_{m} \cap D^{c}}\right)_{\text {int } D}^{+} \subset c l_{+}\left(\left(V_{1}, \ldots, V_{m}\right)_{D}^{+}\right)=c l_{+}\left(f\left(\mathbf{U}_{+}\right)\right) \subset$ $\mathrm{U}_{+}$. In virtue of Lemma $2.4 \overline{D^{c}} \subset B^{c}$; further $V_{1}, \ldots, V_{m}$ can be chosen so that $\overline{V_{j} \cap D^{c}} \subset U_{j} \cap B^{c}$ for all $1 \leq j \leq m$. Then by Lemma 2.3(i) we have

$$
\begin{aligned}
c l_{*}\left(f_{*}(\mathbf{U})\right) & =c l_{*}\left(\left(V_{1}, \ldots, V_{m}\right)_{D}^{*}\right)=\left(\overline{V_{1} \cap D^{c}}, \ldots, \overline{V_{m} \cap D^{c}}\right)_{i n t D}^{*} \\
& =\left(\left(\overline{V_{1} \cap D^{c}}, \ldots, \overline{V_{m} \cap D^{c}}\right)_{i n t D}^{+}\right)_{*} \subset\left(\mathbf{U}_{+}\right)_{*}=\mathbf{U},
\end{aligned}
$$

hence $f_{*} \in R S\left(X^{\omega}, \mathcal{B}_{\Delta}^{*}\right)$. Finally (iii) follows analogously from Lemma 2.3(iii) and Lemma 2.4 .

\section{Quasi-REgularity of (PROXIMAL) HIT-AND-MisS TOPOLOGIES}

We will say that $\Delta \subset C L(X)$ is a quasi-Urysohn (uniformly quasi-Urysohn) family provided whenever $B \in \Sigma(\Delta)$ and $W_{i} \in \tau \backslash\{\emptyset\}$ are disjoint for each $1 \leq i \leq n$ $(n \in \omega)$, there exists $D \in \Sigma(\Delta)$ such that $B \subset$ int $D \subset D \quad(U[B] \subset D$ for some $U \in \mathcal{U}$ ) and $W_{i} \cap D^{c} \neq \emptyset$ for every $1 \leq i \leq n$. We will say that $X$ is $\Delta$-quasi-regular provided whenever $W \in \tau \backslash\{\emptyset\}$ there exists $B \in \Delta$ such that $\emptyset \neq$ int $B \subset B \subset W$. If $\Delta=C L(X)$ this reduces to quasi-regularity of $X$ and for $\Delta=K(X)$ we get socalled almost locally compact spaces (cf. [MCN]). Further $X$ will be called uniformly $\Delta$-quasi regular provided whenever $\emptyset \neq W$ is an open subset of $X$ there exists $B \in \Delta$ and $U \in \mathcal{U}$ with $U[B] \subset W$. This condition holds for $\Delta=C L(X), K(X)$ and $B(X)$.

Theorem 3.1. (i) If $\left(C L(X), \tau_{\Delta}^{+}\right)$(resp. $\left(C L(X), \tau_{\Delta}^{++}\right)$) is quasi-regular, then $\Delta$ is a (uniformly) quasi-Urysohn family and $X$ is quasi-regular. Further in both cases $\left(X^{\omega}, \tau_{\Delta}^{*}\right)$ is quasi-regular. 
(ii) If $X$ is (uniformly) $\Delta$-quasi-regular and $\Delta$ is a (uniformly) quasi-Urysohn family, then $\left(C L(X), \tau_{\Delta}^{+}\right)$(resp. $\left.\left(C L(X), \tau_{\Delta}^{++}\right)\right)$is a quasi-regular space.

Proof. (i) Suppose that there exists a non-pseudo- $R_{0} X$ such that $\left(C L(X), \tau_{\Delta}^{+}\right)$is quasi-regular. Then there exists a nonempty $\tau$-open $V$ with $V^{+}=\emptyset$ and we can find $A \in V^{-}$and $\mathbf{V} \in \mathcal{B}_{\Delta}^{+}$such that $A \in \mathbf{V} \subset c l_{+}(\mathbf{V}) \subset V^{-}$. Then similarly to Lemma 1 in [Zs] it follows that $A \cap V^{c} \in c l_{+}(\{A\}) \subset c l_{+}(\mathbf{V}) \subset V^{-}$, which is a contradiction. So $X$ is pseudo- $R_{0}$. Now if $U \in \tau \backslash\{\emptyset\}$, then the quasi-regularity of $\tau_{\Delta}^{+}$yields a nonempty $\left(V_{1}, \ldots, V_{m}\right)_{S} \in \mathcal{B}_{+}$with $\tau_{\Delta}^{+}$-closure contained in $U^{-}$. In virtue of Lemma 2.3(ii) and Lemma 2.4 we get that $\emptyset \neq \overline{V_{j} \cap S^{c}} \subset U$ for some $1 \leq j \leq m$, which implies the quasi-regularity of $X$. Finally, if $B \in \Sigma(\Delta)$ and $W_{i} \in \tau \backslash\{\emptyset\}$ are disjoint for each $1 \leq i \leq k(k \in \omega)$, then there exists a nonempty $\mathbf{U}=\left(U_{1}, \ldots, U_{n}\right)_{D}^{+} \in \mathcal{B}_{\Delta}^{+}$such that $\mathbf{U} \subset c l_{+}(\mathbf{U}) \subset\left(W_{1}, \ldots, W_{k}\right)_{B}^{+}$. According to Lemma 2.3(ii) we have $\overline{D^{c}} \in\left(W_{1}, \ldots, W_{k}\right)_{B}^{+}$, so $\overline{D^{c}} \subset B^{c}$ and $W_{i} \cap \overline{D^{c}} \neq \emptyset$ for all $1 \leq i \leq k$; whence $\Delta$ is a quasi-Urysohn family. The proximal case works out similarly making use of Lemma 2.3(iii) and Lemma 2.4. Finally the quasi-regularity (complete regularity) of $X$ and that $\Delta$ is a (uniformly) quasi-Urysohn family easily imply the quasi-regularity of $\left(X^{\omega}, \tau_{\Delta}^{*}\right)$ by Lemma 3.3(i).

(ii) Take a nonempty $\mathbf{U}=\left(U_{1}, \ldots, U_{n}\right)_{B}^{+} \in \mathcal{B}_{\Delta}^{+}$. We can choose $D \in \Sigma(\Delta)$ such that $B \subset$ int $D \subset D$ and $U_{i} \cap D^{c} \neq \emptyset$ for all $1 \leq i \leq n$. Further pick $S_{i} \in \Delta$ with $\emptyset \neq i n t S_{i} \subset S_{i} \subset U_{i} \cap D^{c}$, and $x_{i} \in$ int $S_{i}$ with $\overline{\left\{x_{i}\right\}} \subset i n t S_{i}$ for every $1 \leq i \leq n$. Put $\mathbf{V}=\left(\text { int } S_{1}, \ldots, \text { int } S_{n}\right)_{D}^{+}$. Then $\bigcup_{i=1}^{n} \overline{\left\{x_{i}\right\}} \in \mathbf{V}$ and if $C \in c l_{+}(\mathbf{V}) \backslash \mathbf{U}$, then either $C \nsubseteq B^{c}$ or $C \cap U_{i}=\emptyset$ for some $1 \leq i \leq n$. In the first case $C \in(\text { int } D)^{-}$and in the second $C \in\left(S_{i}^{c}\right)^{+}$, contradicting $C \in c l_{+}(\mathbf{V})$. It means that $\emptyset \neq \mathbf{V} \subset c l_{+}(\mathbf{V}) \subset \mathbf{U}$, so $\left(C L(X), \tau_{\Delta}^{+}\right)$is quasi-regular. The proximal case works similarly. Indeed, if now $\mathbf{U}=\left(U_{1}, \ldots, U_{n}\right)_{B}^{++} \in \mathcal{B}_{\Delta}^{++}$, then we can choose $D \in \Sigma(\Delta)$ and $U \in \mathcal{U}$ with $U[B] \subset D$ and $U_{i} \cap D^{c} \neq \emptyset$, further $S_{i} \in \Delta$ and $W_{i} \in \mathcal{U}$ with $W_{i}\left[S_{i}\right] \subset U_{i}$ $(1 \leq i \leq n)$. Then $\bigcup_{i=1}^{n} S_{i} \in\left(W_{1}\left[S_{1}\right], \ldots, W_{n}\left[S_{n}\right]\right)_{D}^{++}=\mathbf{V}$ and an argument similar to that for the hit-and-miss case applies.

Remark 1. The necessary conditions for quasi-regularity of the (proximal) hit-andmiss topology in Theorem 3.1(i) are not sufficient conditions. Indeed, consider $X=[0,2]$ with the Euclidean metric, and put $U=[0,1)$ and $\Delta=\{[x, 2] ; x \in$ $(1,2]\}$. Then $\Delta$ is a quasi-Urysohn family; further $U^{c} \cap \overline{B^{c}} \in c l_{+}(\mathbf{U}) \backslash U^{-}$whenever $\mathbf{U}=\left(U_{1}, \ldots, U_{n}\right)_{B}^{+} \in \mathcal{B}_{\Delta}^{+}$. It means that $\left(C L(X), \tau_{\Delta}^{+}\right)$is not quasi-regular. The proximal case works out similarly. Finally, according to Lemma 2.3(i) $\left(X^{\omega}, \tau_{\Delta}^{*}\right)$ is quasi-regular; thus, quasi-regularity for $\tau_{\Delta}^{*}$ does not imply quasi-regularity for $\tau_{\Delta}^{+}$ (resp. $\tau_{\Delta}^{++}$) in general.

Corollary 3.2. (i) $\left(C L(X), \tau_{V}\right)$ is quasi-regular if and only if $X$ is quasi-regular;

(ii) Let $X$ be a uniform space. Then $\left(C L(X), \tau_{p V}\right)$ is quasi-regular.

Proof. (i) Observe that if $\Delta=C L(X)$, then $\Delta$-quasi-regularity of $X$ as well as the quasi-Urysohn property for $\Delta$ coincide with quasi-regularity of $X$. As for (ii) observe that both conditions of Theorem 3.1 follow from complete regularity of the uniform topology.

Corollary 3.3. (i) $\left(C L(X), \tau_{F}\right)$ is quasi-regular if and only if $X$ is almost locally compact and the closed compact subsets of $X$ have open neighbourhoods with compact closure. 
(ii) $\left(C L(X), \tau_{p F}\right)$ is quasi-regular if and only if $X$ is a locally compact uniform space.

Proof. (i) Sufficiency: If $B \in K(X)$ and $U_{i}$ is a nonempty $\tau$-open set disjoint to $B$ for each $1 \leq i \leq n(n \in \omega)$, we can choose a closed compact subset $B_{i}$ of $U_{i}$ with nonempty interior. Further $\overline{\{x\}}$ is compact for each $x \in B$, so there exists a nonempty $\tau$-open neighbourhood $V_{x}$ of $x$ with compact closure such that $\overline{V_{x}} \subset \bigcap_{i=1}^{n} B_{i}^{c}$. Then the compactness of $B$ guarantees a finite family $V_{x_{1}}, \ldots, V_{x_{m}}$ covering $B$, hence choosing $D=\bigcup_{j=1}^{m} \bar{V}_{x_{j}}$ we see that $K(X)$ is a quasi-Urysohn family. Now Theorem 3.1(ii) applies. Conversely, in view of Theorem 3.1(i) it suffices to show that quasi-regularity of the Fell topology forces almost local compactness on $X$. So let $W \in \tau \backslash\{\emptyset\}$. First suppose that some closed compact subset of $X$ meets $W$. Then there exists $K \in K(X)$ with $W \cap i n t K \neq \emptyset(K(X)$ is a quasi-Urysohn family by Theorem 3.1(i)), hence the quasi-regularity of $X$ yields a $K_{1} \in K(X)$ with $\emptyset \neq$ int $K_{1} \subset K_{1} \subset W$. Suppose now that no member of $K(X)$ meets $W$. By the quasi-regularity of $\left(C L(X), \tau_{F}\right)$ we get some nonempty $\mathbf{W}=\left(W_{1}, \ldots, W_{k}\right)_{S}^{+} \in \tau_{F}$ with $\tau_{F^{-}}$-closure contained in $W^{-}$. If $S^{c} \cap W^{c} \neq \emptyset$, then it is not hard to show that $\overline{S^{c} \cap W^{c}} \in \operatorname{cl}_{F}(\mathbf{W}) \subset W^{-}$, which is a contradiction. Consequently $S^{c}=W$ and $W$ has no nonempty open subset different from $W$. Accordingly $W$ is compact and from quasi-regularity of $X$ we can infer that $W$ is closed as well, which contradicts the choice of $W$.

(ii) It has been already mentioned that a uniform space is always $K(X)$-quasiregular. Further an easy compactness argument yields that local compactness of $X$ implies that $K(X)$ is a uniformly quasi-Urysohn family. Conversely, the uniform topology is $R_{0}$, so point-closures are compact; thus applying the uniform quasiUrysohn property for point-closures we get local compactness of $X$.

Corollary 3.4. Let $(X, d)$ be a metric space. Then $\left(C L(X), \tau_{B}\right)$ and $\left(C L(X), \tau_{p B}\right)$ are quasi-regular.

Proof. Let $B=\bigcup_{j=1}^{m} B\left(x_{j}, \alpha_{j}\right) \in \Sigma(B(X))$ and $W_{i}=S\left(y_{i}, \beta_{i}\right)$ be disjoint for each $1 \leq i \leq n\left(y_{i} \in X, \beta_{i}>0\right)$. Then $\gamma_{j}=\min _{1 \leq i \leq n} d\left(x_{j}, y_{i}\right)>\alpha_{j}$ for every $1 \leq j \leq$ $m$, so choosing $D=\bigcup_{j=1}^{m} B\left(x_{j}, \frac{\alpha_{j}+\gamma_{j}}{2}\right)$ we easily get that $B(X)$ is a (uniformly) quasi-Urysohn family. As for (uniform) "ball"-quasi-regularity it suffices for any $W=S(x, \alpha)$ to pick $B=B\left(x, \frac{\alpha}{2}\right)$.

Remark 2. Note here that the ball and the ball-proximal topology need not be regular in general (see [HL] and [H], Theorem 4.1).

\section{Baire SPACES AND (PROXIMAL) HIT-AND-MisS TOPOLOGIES}

By a proper modification of the proof of Theorem 3.8 in [MC] we can prove our main theorem.

Theorem 4.1. Suppose that $\left(X^{\omega}, \tau_{\Delta}^{*}\right)$ is a Baire space and $\left(C L(X), \tau_{\Delta}^{+}\right)$(respectively $\left.\left(C L(X), \tau_{\Delta}^{++}\right)\right)$is quasi-regular. Then $\left(C L(X), \tau_{\Delta}^{+}\right)\left(\right.$resp. $\left.\left(C L(X), \tau_{\Delta}^{++}\right)\right)$is a Baire space.

Proof. Let $\mathbf{U} \in \mathcal{B}_{\Delta}^{+}$and $f \in R S\left(C L(X), \mathcal{B}_{\Delta}^{+}\right)$. Then by Theorem 3.1(i) $X$ is pseudo- $R_{0}$, so by Lemma 2.5(ii) $f_{*} \in R S\left(X^{\omega}, \mathcal{B}_{\Delta}^{*}\right)$. Further in view of Theorem 3.1(i) $\left(X^{\omega}, \tau_{\Delta}^{*}\right)$ is a quasi-regular Baire space; thus according to Theorem 2.1 there exists $g \in S\left(X^{\omega}, \mathcal{B}_{\Delta}^{*}\right)$ such that $\bigcap_{i=1}^{\infty}\left[\mathbf{U}_{*}, f_{*}, g\right]_{i}$ contains some element $\left(x_{k}\right)_{k} \in X^{\omega}$. 
It can be shown that $\left[\mathbf{U}, f, g_{+}\right]_{2 n}=\left(\left[\mathbf{U}_{*}, f_{*}, g\right]_{2 n}\right)_{+}$for all $n \in \omega$ (cf. [MC],Theorem 3.8). Observe that by Lemma $2.5(\mathrm{i}) g_{+} \in S\left(C L(X), \mathcal{B}_{\Delta}^{+}\right)$. Now whenever $n>1$, then $\left(x_{k}\right)_{k} \in\left[\mathbf{U}_{*}, f_{*}, g\right]_{2 n}=\left(U_{1}, \ldots, U_{m}\right)_{B}^{*}$ for some $U_{1}, \ldots, U_{m} \in \tau, B \in \Sigma\left(\Delta_{0}\right)$ and $m \in \omega$. Consequently $x_{k} \in B^{c}$ for all $k \in \omega$ and $x_{k} \in U_{k} \cap B^{c}$ for each $1 \leq k \leq m$. Then $A=\overline{\left\{x_{k} ; k \in \omega\right\}} \subset \overline{B^{c}}$ and $A \cap\left(U_{k} \cap B^{c}\right) \neq \emptyset$ for every $1 \leq k \leq m$, thus in virtue of Lemma 2.3(ii) we have

$$
\begin{gathered}
A \in\left(U_{1} \cap B^{c}, \ldots, U_{m} \cap B^{c}\right)_{\text {intB }}^{+} \subset c l_{+}\left(\left(U_{1}, \ldots, U_{m}\right)_{B}^{+}\right) \\
=c l_{+}\left(\left(\left[\mathbf{U}_{*}, f_{*}, g\right]_{2 n}\right)_{+}\right)=c l_{+}\left(\left[\mathbf{U}, f, g_{+}\right]_{2 n}\right)=c l_{+}\left(f\left(g_{+}\left(\left[\mathbf{U}, f, g_{+}\right]_{2 n-2}\right)\right)\right) \\
\subset g_{+}\left(\left[\mathbf{U}, f, g_{+}\right]_{2 n-2}\right) \subset\left[\mathbf{U}, f, g_{+}\right]_{2 n-2}
\end{gathered}
$$

for every $n>1$. Accordingly $\bigcap_{i=1}^{\infty}\left[\mathbf{U}, f, g_{+}\right]_{i} \neq \emptyset$, so $\left(C L(X), \tau_{\Delta}^{+}\right)$is a Baire space by Theorem 2.1 .

Finally an analogous argument justifies the theorem for the proximal hit-andmiss topology as well. It suffices only to use Lemma 2.5(iii) instead of Lemma 2.5(ii) and Lemma 2.3(iii) instead of Lemma 2.3(ii).

Corollary 4.2. Let $X$ be a (uniformly) $\Delta$-quasi-regular Baire space with a countable pseudo-base. Suppose that $\Delta$ contains a countable (uniformly) quasi-Urysohn family $\Delta^{\prime}$ (i.e. in the definition of (uniformly) quasi-Urysohn family we require $\left.D \in \Sigma\left(\Delta^{\prime}\right)\right)$.

Then $\left(C L(X), \tau_{\Delta}^{+}\right)$(resp. $\left.\left(C L(X), \tau_{\Delta}^{++}\right)\right)$is a Baire space.

Proof. See Lemma 2.2, Theorem 3.1(ii) and Theorem 4.1.

In the sequel we will adopt the informal definition of complete spaces (cf. [FK]), i.e. spaces that can be proved Baire by an argument similar to the proof of the Baire Category Theorem. Examples of such spaces are e.g. the completely metrizable spaces, locally compact regular (Hausdorff) spaces, pseudo-complete spaces, Čechcomplete spaces or the (weakly) $\alpha$-favourable spaces (see [HMC], [AL], [W]).

Theorem 4.3. Suppose that $X$ is a complete space and $\left(C L(X), \tau_{\Delta}^{+}\right)((C L(X)$, $\left.\left.\tau_{\Delta}^{++}\right)\right)$is quasi-regular. Then $\left(C L(X), \tau_{\Delta}^{+}\right)\left(\left(C L(X), \tau_{\Delta}^{++}\right)\right)$is a Baire space.

Proof. It can be shown similarly to $[\mathrm{FK}]$ (p. 230) using Lemma 2.3(i) that the completeness of $X$ implies that $\left(X^{\omega}, \tau_{\Delta}^{*}\right)$ is a Baire space. Further use Theorem 4.1 .

Corollary 4.4. Let $X$ be a quasi-regular (uniform) space. If $X$ is complete or is a Baire space having a countable pseudo-base, then $\left(C L(X), \tau_{V}\right)\left(\left(C L(X), \tau_{p V}\right)\right)$ is a Baire space.

Proof. See Corollary 3.2, Corollary 4.2 and Theorem 4.3.

Remark 3. Notice that the non-parenthetic part of the previous corollary is precisely the result stated in $[\mathrm{MC}]$. However less is actually proved, since the proof of Corollary 3.9 in $[\mathrm{MC}]$ implicitly uses that $X$ is further a $T_{1}$-space.

Corollary 4.5. (i) If $X$ is almost locally compact and the closed compact subsets of $X$ have open neighbourhoods with compact closure, then $\left(C L(X), \tau_{F}\right)$ is a Baire space.

(ii) Let $X$ be a locally compact uniform space. Then $\left(C L(X), \tau_{p F}\right)$ is a Baire space. 
Proof. (i) In view of Corollary 3.3(i) $\left(C L(X), \tau_{F}\right)$ is quasi-regular. Observe that the classical proof of Bairness of locally compact regular spaces (cf. $[\mathrm{K}]$, Theorem 34 , p. 200) works for almost locally compact spaces as well; thus, $X$ is complete and Theorem 4.3 applies. Case (ii) can be shown similarly using Corollary 3.3(ii) instead of Corollary 3.3(i).

Remark 4. The above corollary is interesting only for non-regular or non-locally compact spaces, since otherwise the Fell topology makes $C L(X)$ a locally compact Hausdorff space (see $[\mathrm{KT}]$ and $[\mathrm{Zs}]$, Theorem 2), hence the hyperspace is a Baire space. On the other hand Example 3.4 in $[\mathrm{MCN}]$ demonstrates that conditions of Corollary 4.5(i) are more general than local compactness plus regularity.

Corollary 4.6. Let $X$ be a metric space. If $X$ is a separable Baire space or is complete, then $\left(C L(X), \tau_{B}\right)$ as well as $\left(C L(X), \tau_{p B}\right)$ is a Baire space.

Proof. If $X$ is complete, then Theorem 4.3 and Corollary 3.4 apply. Suppose now that $(X, d)$ is a separable Baire metric space and $E$ is a countable dense subset of $X$. Then $\Delta^{\prime}=\{B(e, r) \in B(X) ; e \in E, r$ is a positive rational $\}$ is a countable subfamily of $\Delta=B(X)$. If $B=\bigcup_{j=1}^{m} B\left(x_{j}, \alpha_{j}\right) \in \Sigma(B(X))$ and $F \neq \emptyset$ is a finite subset of $B^{c}$, then $\beta_{j}=d\left(x_{j}, F\right)-\alpha_{j}>0$; further there exists $e_{j} \in E \cap S\left(x_{j}, \frac{\beta_{j}}{4}\right)$ and a rational $r_{j}$ such that $\alpha_{j}+\frac{\beta_{j}}{4}<r_{j}<d\left(x_{j}, F\right)-\frac{\beta_{j}}{4}$ for each $1 \leq j \leq m$. Then $B\left(x_{j}, \alpha_{j}\right) \subset B\left(e_{j}, r_{j}\right)$ for all $1 \leq j \leq m$ and $F \subset D^{c}$ where $D=\bigcup_{j=1}^{m} B\left(e_{j}, r_{j}\right) \in$ $\Sigma\left(\Delta^{\prime}\right)$; thus, $\Delta^{\prime}$ satisfies conditions of Lemma 2.2(i). Consequently $\left(X^{\omega}, \tau_{B(X)}^{*}\right)$ is a Baire space and Theorem 4.1 applies.

\section{Baire SPACES AND the WiJsman topology}

It is well known that for certain base spaces $\tau_{p B}=\tau_{W}$, e.g. it holds in any normed linear space (cf. [HL] for conditions characterizing this equality). As the following theorem demonstrates there is an even closer connection between $\tau_{p B}$ and $\tau_{W}$ as far as their Baireness is concerned.

Theorem 5.1. $\left(C L(X), \tau_{W}\right)$ is a Baire space if and only if $\left(C L(X), \tau_{p B}\right)$ is.

Proof. The identity $I:\left(C L(X), \tau_{p B}\right) \rightarrow\left(C L(X), \tau_{W}\right)$ is continuous since $\tau_{W} \subset \tau_{p B}$ ([B2], Theorem 2.2.3(a)). Now choose any nonempty $\mathbf{U}=\left(U_{1}, \ldots, U_{n}\right)_{B}^{++} \in \mathcal{B}_{B(X)}^{++}$, where $B=\bigcup_{j=1}^{m} B_{j}, B_{j}=B\left(x_{j}, \alpha_{j}\right) \quad\left(x_{j} \in X, \alpha_{j}>0,1 \leq j \leq m\right)$. If $A_{0} \in \mathbf{U}$, then $A_{0} \cap U_{i} \neq \emptyset(1 \leq i \leq n)$ and $D\left(B, A_{0}\right)>0$, so there exists $a_{i} \in A_{0} \cap U_{i}$ with $d\left(x_{j}, a_{i}\right)>\alpha_{j}$ for all $1 \leq i \leq n$ and $1 \leq j \leq m$. Denote $A_{1}=\left\{a_{1}, \ldots, a_{n}\right\}$ and put $\alpha=\min _{1 \leq j \leq m} d\left(x_{j}, A_{1}\right)$. Then $\alpha>\alpha_{j}$ for all $1 \leq j \leq m$, so defining $\mathbf{U}_{0}=\bigcap_{j=1}^{m}\left\{A \in C L(X) ; d\left(x_{j}, A\right)>\frac{\alpha+\alpha_{j}}{2}\right\} \cap \bigcap_{i=1}^{n} U_{i}^{-} \in \tau_{W}$, we have $A_{1} \in \mathbf{U}_{0}$. Further if $A \in \mathbf{U}_{0}$, then $D\left(A, B_{j}\right) \geq \frac{\alpha-\alpha_{j}}{2}>0$ for each $1 \leq j \leq m$. It means that $\emptyset \neq \mathbf{U}_{0} \subset \mathbf{U}$, whence $I$ is a feeble homeomorphism and such mappings preserve Baire spaces (see $[\mathrm{N}]$ ).

Corollary 5.2. If $X$ is a separable Baire metric space or is a complete space, then $\left(C L(X), \tau_{W}\right)$ is a Baire space.

Proof. See Theorem 5.1 and Corollary 4.6.

Remark 5. It is worth noticing in connection with Corollary 5.2 that for every separable completely metrizable space $X$ the Wijsman topology is completely metrizable 
(cf. [B1], [C]). On the other hand, Costantini constructed a complete metric space $X$ such that $\left(C L(X), \tau_{W}\right)$ is not Cech-complete.

\section{REFERENCES}

[AL] J.M.Aarts-D.J.Lutzer, Completeness properties designed for recognizing Baire spaces, Dissertationes Math. 116 (1974), 1-48. MR 52:1642

[B1] G.Beer, A Polish topology for the closed subsets of a Polish space, Proc. Amer. Math. Soc. 113 (1991), 1123-1133. MR 92c:54009

[B2] G.Beer, Topologies on Closed and Closed Convex Sets, Kluwer, Dordrecht, 1993. MR 94:10

[BLLN] G.Beer-A.Lechicki-S.Levi-S.Naimpally, Distance Functionals and Suprema of Hyperspace Topologies, Annali Mat. Pura Appl. 162 (1992), 367-381. MR 94c:54016

[BT1] G.Beer-R.Tamaki, On hit-and-miss hyperspace topologies, CMUC 34 (1993), 717-728. MR 95a:54022

[BT2] G.Beer-R.Tamaki, The Infimal Value Functional and the Uniformization of Hit-andMiss Hyperspace Topologies, Proc. Amer. Math. Soc. 122 (1994), 601-612. MR 95a:54023

[C] C.Costantini, Every Wijsman topology relative to a Polish space is Polish, Proc. Amer. Math. Soc. 123 (1995), 2569-2574. CMP 94:11

[CLP] C.Costantini-S.Levi-J.Pelant, Infima of hyperspace topologies, Mathematica 42 (1995), 67-86.

[D] A.Davis, Indexed systems of neighbourhoods for general topological spaces, Amer. Math. Monthly 68 (1961), 886-893. MR 35:4869

[DMH] G.Di Maio-Ľ.Holá, On hit-and-miss topologies, preprint.

[DMN] G.Di Maio-S.Naimpally, Comparison of hypertopologies, Rend. Instit. Mat. Univ. Trieste 22 (1990), 140-161. MR 94b:54029

[F] J.Fell, A Hausdorff topology for the closed subsets of locally compact non-Hausdorff space, Proc. Amer. Math. Soc. 13 (1962), 472-476. MR 52:2573

[FK] W.G.Fleissner-K.Kunen, Barely Baire spaces, Fund. Math. 101 (1978), 229-240. MR 80f:54009

[FLL] S.Francaviglia-A.Lechicki-S.Levi, Quasi-uniformization of hyperspaces and convergence of nets of semicontinuous multifunctions, J. Math. Anal. Appl. 112 (1985), 347-370. MR 87e:54025

$[\mathrm{H}] \quad \check{L} . H o l a ́, W h e n$ Wijsman topology is the weak topology generated by gap functionals determined by closed proper balls, preprint

[HL] Ľ.Holá-R.Lucchetti, Equivalence among hypertopologies, Set-Valued Analysis 3 (1995), 339-350. CMP 96:07

[HMC] R.C.Haworth-R.A.McCoy, Baire spaces, Dissertationes Math. 141 (1977), 1-77. MR 55:4106

[K] J.L.Kelley, General Topology, Springer-Verlag, New York, 1984.

[KT] E.Klein-A.Thompson, Theory of Correspondences, Wiley, New York, $1975 . \quad$ MR 86a:90012

[MC] R.A.McCoy, Baire spaces and hyperspaces, Pacific J. Math. 58 (1975), 133-142. MR 53:14436

[MCN] R.A.McCoy-I.Ntantu, Properties of $C(X)$ with the Epi-Topology, Boll. Un. Mat. Ital. (7) 6-7 (1992), 507-532. MR 94e:54020

[Mi] E.Michael, Topologies on spaces of subsets, Trans. Amer. Math. Soc. 71 (1951), 152-182. MR 13:54f

[N] T.Neubrunn, A note on mappings of Baire spaces, Math. Slovaca 27 (1977), 173-176. MR 56:13153

[O] J.C.Oxtoby, Cartesian products of Baire spaces, Fund. Math. 49 (1961), 157-166. MR 25:4055

[P1] H.Poppe, Eine Bemerkung über Trennungsaxiome in Räumen von abgeschlossenen Teilmengen topologisher Räume, Arch. Math. 16 (1965), 197-199.

[P2] H.Poppe, Einige Bemerkungen über den Raum der abgeschlossenen Mengen, Fund. Math. 59 (1966), 159-169. MR 33:6573 
[V] W.Vervaat, Random upper semicontinuous functions and extremal processes, Centre for Mathematics and Computer Science, Report MS-R8801, Amsterdam, 1988.

[W] H.E.White, Jr., Topological spaces that are $\alpha$-favourable for a player with perfect information, Proc. Amer. Math. Soc. 50 (1975), 477-482. MR 51:4183

[Zs] L.Zsilinszky, On separation axioms in hyperspaces, Rend. Circ. Mat. Palermo 45 (1996), $75-83$.

Department of Mathematics, University of Ostrava, Bráfova 7, 70103 Ostrava, Czech Republic

E-mail address: zsilin@oudec.osu.cz

Current address: Department of Mathematics, University of South Carolina, Columbia, South Carolina 29208

E-mail address: zsilinsz@math.sc.edu 